\title{
On Energy Reduction and Green Networking Enhancement due to In-Network Caching
}

\author{
Junji Takemasa*, Yuki Koizumi*, Toru Hasegawa*, and Ioannis Psaras ${ }^{\dagger}$ \\ ${ }^{*}$ Graduate School of Information Science and Technology, Osaka University, Japan \\ Email: \{j-takemasa, ykoizumi, t-hasegawa\}@ist.osaka-u.ac.jp \\ ${ }^{\dagger}$ Department of Electrical and Electronic Engineering, University College London, UK \\ Email: i.psaras@ucl.ac.uk
}

\begin{abstract}
In-network caching in information centric networking (ICN) is considered as a promising approach to reducing energy consumption of an entire network. However, it is also considered as an energy consuming technique. These contradictory claims lead to one research question: Does caching really reduce the energy consumption of the entire network? To answer the question, we formulate an ICN network as an optimization problem with a realistic energy consumption model for an ICN router. By solving the formulation assuming that ICN forwarding software currently under development is used as a forwarding engine of an ICN router, we reveal that in-network caching alone does not reduce much energy but it enhances a currently developed green networking technique even though the forwarding engine is not fully optimized.
\end{abstract}

\section{INTRODUCTION}

On the one hand in-network caching in information centric networking (ICN) is considered as one of promising approaches to reducing energy consumption of an entire network [1], [2], but on the other hand it is considered as a somewhat energy consuming technique [3], [4].

In [4], the authors reveal that in-network caching has a negative effect on energy consumption. We simply refer to in-network caching as caching, hereafter. They empirically model the energy consumption of an ICN software router and show that name-based packet forwarding and packet-level caching are energy consuming. Though energy reduction with caching is a hot research topic [1], [2], many studies focus on traffic reduction due to caching, which may reduce energy as a byproduct, without considering the increase in energy consumption due to name-based packet forwarding and packetlevel caching.

From the findings in [4], the following research question arises: Does caching really reduce the energy consumption of the entire network? To answer this question, we make two hypotheses that i) caching alone does not reduce energy consumption of an entire network but ii) it enhances currently developed green networking techniques. The hypothesis i) is rather straightforward because of the trade-off relation of caching on the energy consumption. The hypothesis ii) comes from the fact that caching reduces traffic in the entire network [2] and the performance of many green networking techniques, in general, depends on traffic load as discussed in their studies [5].
In this paper, we first analyze energy reduction effects of caching alone focusing on the energy decreased by the traffic reduction and increased by name-based packet forwarding. Next, we integrate caching and a currently developed green networking technique and how the traffic reduction effect owing to caching affects the green networking technique. As a candidate of green networking techniques that can be used in conjunction with caching, we use an energy-aware routing [5], which aggregates traffic on several links and cuts off the power of unused links. To investigate the potential energy reduction effects, we model these green networking techniques as optimization problems with an objective function to minimize energy consumption. Then, we derive the lower bounds of their consumed energy.

The contributions of this paper are two-fold. First, we formulate green networking techniques with a realistic energy consumption model of an ICN router [4]. Second, by solving the formulation assuming that ICN forwarding software currently under development is used as a forwarding engine of an ICN router, we reveal that how caching alone reduces the energy consumption and how caching enhances the energy reduction effects of energy-aware routing.

The rest of this paper is organized as follows. We introduce green networking techniques in Section II. We describe our target network in Section III and formulate the green networking techniques as optimization problems in Section IV. Section V shows numerical results. In Section VI, we briefly introduce the related work. Finally, Section VII concludes this paper.

\section{GREEN NETWORKING}

\section{A. Caching}

1) Benefit and Drawback of Caching: We summarize a benefit and a drawback of caching for energy consumption.

Benefit: The main factor of caching on energy reduction is to reduce traffic on routers. Because of caching, requests for contents need not traverse the entire network toward servers that originate contents. Thus, it reduces energy consumed for packet processing at upstream routers [2].

Drawback: However, complex packet processing for caching imposes high energy consumption at routers [4].

Though caching is considered as one of green networking techniques [1], [2], these studies do not consider the drawback. 
The contribution of caching to the energy reduction strongly depends on the trade-off relation of the benefit and drawback.

2) Design Aspects for Caching: The effects of the abovementioned benefit and drawback depend on how caching is designed. Caching has the following three design aspects: i) Cache Placement, which determines which router has caching functionality, ii) Content Placement, which determines what contents are placed on the cache of each router, and iii) Content-level Routing, which determines how requests are routed to cached contents or servers that originate contents. Several heuristic approaches are proposed. However, we derive the optimal performance on energy reduction by solving an optimization problem since our analyses are aimed at investigating the potential energy reduction effects of caching rather than that due to a specific heuristic approach.

\section{B. Energy-aware Routing}

As a currently developed green networking technique, we focus on energy-aware routing [5], which frees several links by moving their traffic onto other links so that the links without traffic can be turned off. This technique includes two control aspects, routing and cutting off links, which determines which link to be cut off. Since caching also has routing control, we refer to the energy-aware routing as cutting to avoid confusion.

\section{Integration of Caching and Energy-aware Routing}

To investigate how the traffic reduction effect due to caching enhances cutting, we integrate caching and cutting. The integrated control includes the above-mentioned design aspects, cache placement, content placement, content-level routing, and cutting off links.

\section{TARGET NETWORK}

\section{A. Overview}

We assume that the whole network consists of several access networks interconnected via point of presences (PoPs). We refer to the PoP-level network as a core network. We focus on both an access network and a core network because of an expectation that caching may work better in an access network than a core network [6] while cutting may work better in a core network than an access network [5]. The performance metric discussed in [6] is not the energy consumption but performance-oriented metric, such as latency. Thus, it is still an open issue whether caching is more effective in terms of the energy reduction in an access network than a core network.

\section{B. Physical Topology}

One of the mainstream communication technologies for network infrastructures is optical networking. For instance, Abilene network uses an optical network that consists of optical add-drop multiplexers (OADMs) and optical fibers as its infrastructure [7]. Thus, we employ the same type of optical networks in this paper.

As physical topologies for an access network and a core network, we assume hierarchically connected optical rings and an optical mesh network, respectively. Figure 1 illustrates an

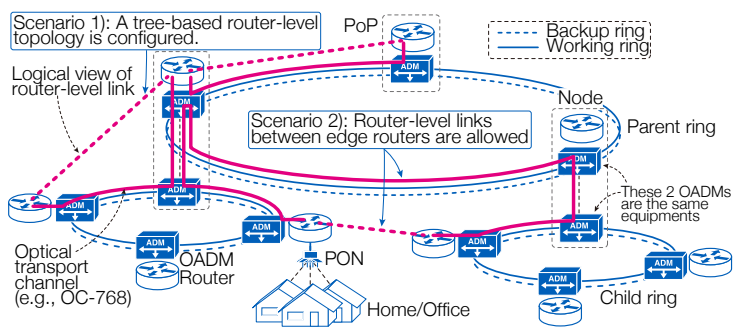

Fig. 1: An example of an access network that consists of hierarchical optical metro rings

example of an access network. Each network consists of nodes having ICN/IP routers overlaying OADMs, with the nodes interconnected by optical fibers. OADMs allow pre-determined optical signals to be added/dropped from/to ICN/IP routers or to be passed through. In such networks, ICN/IP routers are connected with dedicated virtual circuits, i.e., end-to-end optical transport channels. These optical transport channels and ICN/IP routers form a logical topology. We refer to the logical topology and optical transport channels as a routerlevel topology and router-level links, respectively.

Those optical networks consist of three layers, i.e., the ICN, IP, and optical layers. Our target is the ICN layer, including the underlying IP layer, rather than the optical layer because of the fact that ICN/IP routers consume more energy for processing the same amount of traffic than optical devices [4], [8].

\section{Router-level Topology}

It is naturally expected that the energy reduction due to caching and cutting depends on router-level topologies. Therefore, we have to select them carefully. Router-level topologies are determined by configurations of router-level links, i.e., where to establish optical transport channels by configuring OADMs. In this paper, we consider two scenarios about routerlevel topologies: 1) one is the current networks' router-level topologies and 2) another is router-level topologies that are extended by allowing additional router-level links. The current IP access network employs a tree-based topology. Routerlevel topologies of several core networks are opened to the public [7]. We investigate the energy consumption of caching in the scenario 1). Furthermore, to investigate the potential energy reduction effects of caching and cutting, we consider the scenario 2), where several additional 1-hop longer routerlevel links are allowed. For instance, we place router-level links between edge routers for an access network, as shown in Fig. 1. Note that those additional router-level links do not require any modification to the underlying optical network.

\section{FORMUlation}

\section{A. Overview of Formulation}

We formulate 4 models, i.e., i) integration, which includes cache placement, content placement, routing, and cutting off unused links, ii) caching, which includes cache placement, content placement and routing, iii) cutting, which includes 
routing and cutting off links, and iv) no-control, which does not control anything. Due to space limitations, we show only the formulation for integration here. The model for integration has four design variables:

1) $x_{i j} \in\{0,1\}$ is the state of link $(i, j) . x_{i j}=0$ if link $(i, j)$ is sleep, and 1 otherwise.

2) $w_{c, i j}^{u v}$ indicates the Interest packet rate on link $(i, j)$ for content $c$ that goes from router $u$ to $v$.

3) $y_{v} \in\{0,1\}$ indicates cache placement at router $v$. If $y_{v}=1$ if the router $v$ has cache functionality, and 0 otherwise.

4) $z_{v, c} \in\{0,1\}$ indicates content placement. $z_{v, c}$ is 1 if the content $c$ is cached at router $v$.

Note that the model that includes only the variables 1 and 2 corresponds to cutting and that with the variables 2,3 , and 4 does caching. In the case of no-control, no router has cache functionality, $y_{v}=0(\forall v)$, and all traffic is routed on their shortest paths. We use capital and small letters to express constants and variables, respectively.

Our formulation consists of the main formulation part and two sub formulation parts for deriving traffic in caching network and energy consumption. The main formulation contains inequality constraints in our formulation and it is mostly based on the existing optimization problems for routing, as summarized below:

$$
\begin{array}{cll}
\text { minimize } & \sum_{v \in V} e_{v} & \\
\text { subject to } & C_{i j} x_{i j} \geq \lambda_{i j}, & \forall i, j \in V \\
& x_{i j} \leq A_{i j}, & \forall i, j \in V \\
& x_{i j}=x_{j i}, & \forall i, j \in V
\end{array}
$$

The objective function (1) minimizes the total energy consumption of the entire network, where $e_{v}$ is the energy consumed by router $v$, where $V$ is a set of all routers.

The constraint (2) states that a link $(i, j)$ can be turned off if and only if no traffic is routed on it. At the same time, (2) also states that traffic volume on link $(i, j), \lambda_{i j}$, never exceeds its capacity $C_{i j}$. The constraint (3) indicates the existence of an optical layer transport channel between router $i$ and $j$, i.e., link $(i, j)$ cannot be established if there is no underlying optical layer transport channel. This condition is given as a parameter $A_{i j}$, where $A_{i j}=1$ if an underlying optical layer transport channel exists between router $i$ and $j$, and 0 otherwise. The constraint (4) is for establishing fullduplex links between routers. The constraint (5) is for masking a single link or router failure, that is, (5) ensures that all routers have at least one alternative path. Equations (6) and (7) are constraints about content and cache placements, respectively. Equation (6) is the constraint that the destination of Interest packets must be either a router that has the cached content or the content server, where a variable $\beta_{c}^{u v}$ is a fraction of Interest packet for a content $c$ that originates router $u$ and destines for router $v$. Since $\beta_{c}^{u v}$ is a fraction, $\sum_{v \in V} \beta_{c}^{u v}=1(\forall u, \forall c)$ should be satisfied. The existence of the content server is given by the constant $K_{v}$, where $K_{v}=1$ if the content server is connected behind router $v$ and 0 otherwise. The constant $G$ is a set of contents. Equation (7) states 2 constraints, the content $c$ can be cached at router $v$ if it has the cache functionality, $y_{v}$, and the total number of cached contents never exceeds its capacity, $N_{v}$. Finally, (8) is the general flow conservation constraint.

\section{B. Traffic in Caching Network}

Unlike an IP network, where source and destination of packets never change inside the network, those of Interest/Data packets change depending on the cache and content placements in an ICN network. In the ICN network, a client sends an Interest packet to retrieve a content and the Interest packet is forwarded toward a router that caches the content or a content server that originates the content. The router or server that receive the Interest returns the content as a Data packet back to the client. In this section, we describe how traffic in a caching network is formulated.

The Interest packet rate for content $c$ that originates router $u$ and destines for router $v, \lambda_{c}^{u v}$, is expressed as $\lambda_{c}^{u v}=I_{c}^{u} \beta_{c}^{u v}$, where $I_{c}^{u}$ is a rate of Interest packets for $c$ that are originated from clients connecting with $u$. A Data packet is sent backward along the route where the corresponding Interest packet traverses. Thus, traffic volume on link $(i, j)$ is expressed as

$$
\lambda_{i j}=S_{\mathrm{I}} \cdot \sum_{c \in G} \sum_{u \in V} \sum_{v \in V} w_{c, i j}^{u v}+S_{\mathrm{D}} \cdot \sum_{c \in G} \sum_{u \in V} \sum_{v \in V} w_{c, j i}^{u v}
$$

where constant parameters $S_{\mathrm{I}}$ and $S_{\mathrm{D}}$ are the size of Interest and Data packets, respectively.

\section{Router Energy Consumption}

We use an realistic energy consumption model of an ICN router proposed in [4]. In this model, the energy consumed by router $i$ consists of five parts, the energy consumed by line cards (LCs) $\left(e_{i}^{\mathrm{LC}}\right)$, CPUs $\left(e_{i}^{\mathrm{CPU}}\right)$, packet forwarding devices like network interface cards (NICs) $\left(e_{i}^{\mathrm{IP}}\right)$, memory devices $\left(e_{i}^{\mathrm{MEM}}\right)$, and its chassis ( $\left.E_{\mathrm{CHASSIS}}^{\mathrm{ROUTER}}\right)$, and it is expressed as

$$
e_{i}=e_{i}^{\mathrm{LC}}+e_{i}^{\mathrm{CPU}}+e_{i}^{\mathrm{IP}}+e_{i}^{\mathrm{MEM}}+E_{\mathrm{CHASSIS}}^{\mathrm{ROUTER}} .
$$

$E_{\text {CHASSIS }}^{\text {ROUTER }}$ is constant value andother four components are described below.

The energy consumed by LCs, $e_{i}^{\mathrm{LC}}$, is a function of the number of active LCs at router $i$ and it is calculated as,

$$
e_{i}^{\mathrm{LC}}=E^{\mathrm{LC}} \cdot\left\lceil\frac{\sum_{i \in V} x_{i j}}{M_{\mathrm{PORT}}^{\mathrm{LC}}}\right\rceil,
$$

where $E^{\mathrm{LC}}$ is the energy consumed constantly by a LC and $M_{\mathrm{PORT}}^{\mathrm{LC}}$ is the number of ports equipped on the LC. 
The energy consumed by CPUs, $e_{i}^{\mathrm{CPU}}$, is a function of the number of active CPU cores, $n_{i}$, and it is expressed as

$$
e_{i}^{\mathrm{CPU}}=E_{\mathrm{CORE}}^{\mathrm{CPU}} \cdot\left\lceil n_{i}\right\rceil+E_{\mathrm{IDLE}}^{\mathrm{CPU}},
$$

where $E_{\mathrm{CORE}}^{\mathrm{CPU}}$ is the energy consumed by one CPU core and $E_{\mathrm{IDLE}}^{\mathrm{CPU}}$ is that consumed constantly by the CPU board. The number of active CPU cores $n_{i}$ depends on $\lambda_{i}^{\mathrm{IN}}, \lambda_{i}^{\text {OUT }}$, and $\lambda_{i}^{\mathrm{HIT}}$, where $\lambda_{i}^{\mathrm{IN}}$ and $\lambda_{i}^{\mathrm{OUT}}$ are the rates of ingress and egress Interest packets and $\lambda_{i}^{\text {HIT }}$ is the rate of Interest packets that are hit and dropped at router $i$. $\lambda_{i}^{\text {HIT }}$ obviously satisfies $\lambda_{i}^{\mathrm{HIT}}=\lambda_{i}^{\mathrm{IN}}-\lambda_{i}^{\text {OUT }}$ because of the flow conservation principle at routers. $\lambda_{i}^{\mathrm{IN}}$ and $\lambda_{i}^{\mathrm{OUT}}$ is calculated as $\lambda_{i}^{\mathrm{IN}}=\sum_{c \in G}\left(\sum_{u \in V} \sum_{v \in V} \sum_{k \in V} w_{c, k i}^{u v}+I_{i}^{c}\right)$ and $\lambda_{i}^{\text {OUT }}=$ $\sum_{c \in G} \sum_{u \in V} \sum_{v \in V} \sum_{k \in V} w_{c, i k}^{u v}$, respectively. By using $\lambda_{i}^{\mathrm{IN}}$ and $\lambda_{i}^{\text {OUT }}, n_{i}$ is computed as,

$$
n_{i}=\frac{\lambda_{i}^{\mathrm{IN}}\left(H_{1}+y_{i} H_{2}\right)+\lambda_{i}^{\mathrm{HIT}} y_{i} H_{3}+\lambda_{i}^{\mathrm{OUT}}\left(H_{4}+y_{i} H_{5}\right)}{H_{\mathrm{CORE}}},
$$

where $H_{\mathrm{CORE}}$ is the clock speed of the CPU core. The constant $H_{F}$ is clock cycles consumed for processing one Interest or Data packet at a function block $F$ in the ICN software. Thus, the numerator in (13) is the necessary clock cycles per a second for processing incoming packets. The function blocks 2,3 , and 5 are the procedures for caching. Therefore, the clock cycles of those blocks are counted if the router has cache functionality $y_{i}$.

The energy consumed by memory devices, $e_{i}^{\mathrm{MEM}}$, is a function of the memory access rate, $R \cdot \lambda_{i}^{\mathrm{IN}} \cdot S_{\mathrm{D}}$, where $R$ is a constant parameter for converting the rate of ingress packet to the memory access rate. Then, $e_{i}^{\mathrm{MEM}}$ is expressed as,

$$
e_{i}^{\mathrm{MEM}}=E_{\mathrm{BYTE}}^{\mathrm{MEM}} \cdot \lambda_{i}^{\mathrm{IN}} \cdot S_{\mathrm{D}} \cdot R+E_{\mathrm{IDLE}}^{\mathrm{MEM}},
$$

where $E_{\mathrm{BYTE}}^{\mathrm{MEM}}$ and $E_{\mathrm{IDLE}}^{\mathrm{MEM}}$ are the energy consumed for accessing the memory device and its idle time energy consumption.

The energy $e_{i}^{\mathrm{IP}}$ is consumed for processing and forwarding IP packets at network devices such as NICs or route switch processors. This is a function of the IP packet forwarding rate and expressed as,

$$
e_{i}^{\mathrm{IP}}=E_{\mathrm{PACKET}}^{\mathrm{IP}} \cdot \frac{\left(\lambda_{i}^{\mathrm{IN}}+\lambda_{i}^{\mathrm{OUT}}\right)\left(S_{\mathrm{I}}+S_{\mathrm{D}}\right)}{S_{\mathrm{IP}}},
$$

where $S_{\mathrm{IP}}$ is the IP packet size and $E_{\mathrm{PACKET}}^{\mathrm{IP}}$ is the energy consumed for processing one IP packet.

\section{Numerical RESUlT}

\section{A. Evaluation Conditions}

As an access network, we use a hierarchical optical metro ring having 2 child rings and one parent ring as shown in Fig. 1. Each child ring has 3 edge routers, which accommodates traffic from clients. The parent ring has 2 middle routers and 1 core router and each middle router connects the parent and each child ring. Content servers are connected with the core router via one or more core networks. As the core network, we use the Abilene topology [7], where 9 routers constitute an ICN network on top of an optical infrastructure.

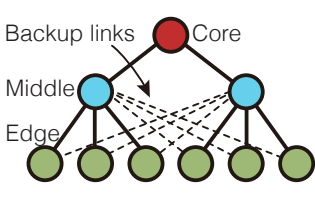

(a) Access network

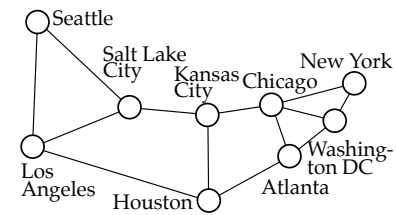

(b) Core network
Fig. 2: Router-level topologies

TABLE I: Parameters for energy consumption model

\begin{tabular}{ll|l|l} 
Parameter & & Software router & Hardware router \\
\hline$E_{\mathrm{CHASSTIS}}^{\mathrm{ROUTER}}$ & {$[\mathrm{J} / \mathrm{s}]$} & 22.56 & 1130 \\
$E_{\mathrm{PACKET}}^{\mathrm{IP}}$ & {$[\mathrm{J} / \mathrm{packet}]$} & $3.04 \times 10^{-6}$ & $1.53 \times 10^{-3}$ \\
$E_{\mathrm{CORE}}^{\mathrm{CPU}}$ & {$[\mathrm{J} / \mathrm{s}]$} & 2.96 & 10 \\
$E_{\mathrm{IDE}}^{\mathrm{CPU}}$ & {$[\mathrm{J} / \mathrm{s}]$} & 2.74 & 0 \\
$H_{\mathrm{CORE}}$ & {$[\mathrm{Hz}]$} & $1.6 \times 10^{9}$ & $2.0 \times 10^{9}$ \\
$E_{\mathrm{BYTE}}^{\mathrm{MEM}}$ & {$[\mathrm{J} / \mathrm{byte}]$} & $0.44 \times 10^{-9}$ & $1.32 \times 10^{-9}$ \\
$E_{\mathrm{IDM}}^{\mathrm{MEM}}$ & {$[\mathrm{J} / \mathrm{s}]$} & 1.1 & 3.3 \\
$E^{\mathrm{LCE}}$ & {$[\mathrm{J} / \mathrm{s}]$} & 27 & 330
\end{tabular}

We place a data center at Atlanta and deploy all content servers at the data center. Figures 2(a) and 2(b) illustrates the routerlevel topologies of the access and core networks. All routers have LCs with a single 40 Gbps port. The core and middle routers in the access network are connected with four links and the other routers are connected with a single link.

In the access network, we deploy ICN software routers, which are suitable for access networks as discussed in [4]. In contrast, we deploy ICN hardware routers in the core network. We draw the constant parameters about the energy consumption of a software ICN router from [4]. For hardware routers, we estimate the values from an ICN hardware router implementation [9]. The router is implemented on a $\mathrm{Cisco}^{\circledR}$ ASR 9006 [10], which has 2 Intel ${ }^{\circledR}$ Xeon ${ }^{\circledR}$ L5638 processors [11] and three times larger DDR3 memory devices than the router in [4]. The idle time energy consumption of the processor is near-zero [11]. The constant $E_{\mathrm{CORE}}^{\mathrm{CPU}}$ is estimated from its thermal design power divided by the number of its cores. Since we have no well-optimized ICN forwarding engine, we assume that both software and hardware routers use CCNx [15], which is currently under development, as their forwarding engines. The clock cycles $H_{F}$ of $\mathrm{CCNx}$ are investigated in [4]. The parameters are summarized in Table I.

Regarding traffic, we use the following conditions. The interest packet rate (packet/s) is determined according to the population of the city. We use the population of cities in Osaka (Japan) [12] and United States [13] for the access network and core network, respectively. We deploy 50 contents in the network. The popularities of the contents are determined according to Zipf distribution with the parameter $\alpha=0.8$ [14]. Cache capacity at each router is set to 5 . We set the size of one Interest packet size to 50 byte, which is estimated on the basis of the URL length of YouTube ${ }^{\mathrm{TM}}$ video contents. We set the Data packet size to 4096 byte, which is the default Data packet size of CCNx. The IP packet size is set to 1500 byte. We use the total content retrieval rate, which is the total 
amount of content size per one second retrieved by all clients.

To get a solution of the formulation within a reasonable time, we made two relaxations. One is linearizing the ceiling function in (12). Another is that we force the solver to stop finding better feasible solutions when it finds a feasible integer solution proved to be within five percent optimal. To check the quality of the feasible solution, we compare it with the optimal integer solution. The error between the objective of the feasible and optimal solutions is less than $0.1 \%$ in the case that total content retrieval rate is $160 \mathrm{Gbps}$ in the access network.

\section{B. Energy Reduction due to Caching}

First, we investigate the effects of caching on the energy consumption without additional router-level links.

Figure 3(a) shows the energy consumption of the entire access network in the case of caching and no-control. The horizontal and vertical axes indicate the energy consumption and the total content retrieval rate, respectively. The result indicates that the energy reduction achieved by caching alone is a little. In the case that the total content retrieval rate is 240 Gbps, caching reduces the energy consumption of the entire network by only $5.1 \%$ compared with no-control while it reduces traffic, i.e., the total number of ingress Interest packets of all routers, by $25.8 \%$. Due to the space limitations, we omit the graph about the total number of ingress Interest packets.

For the more detailed analysis on the energy consumption, we show the energy consumed by each component in routers in the case of the content retrieval rate of $240 \mathrm{Gbps}$ in Fig. 3(b). With caching, the energy consumed by memory and NIC devices decreases. However, the energy consumed by CPUs increases in spite of the decrease in the number of ingress Interest packets. Therefore, the increase in energy consumed by CPUs owing to caching spoils the effect of the energy reduction due to the traffic reduction.

The same tendency is observed in the case of the core network as shown in Fig. 4. In the case that the total content retrieval rate is $120 \mathrm{Gbps}$, caching reduces energy only by $1.3 \%$ compared with no-control while it reduces the total amount of ingress Interest packets by $25.9 \%$. Since the number of packets processed at routers decreases, the energy consumed by memory and NIC devices also decreases by $28.4 \%$. However, the energy consumed by LCs and chassis, which caching can never reduce, occupies the most part of the router energy consumption. Hence, even if we optimally control cache placement, content placement, and content-level routing of caching, caching alone reduces little energy consumption. These results imply that it is difficult to reduce the energy consumption of the entire network with caching alone. In the next section, we discuss how caching enhances cutting.

\section{Enhancing Green Networking}

Next, we employ router-level topology control with wavelength routing. For both the access and core network cases, we assume that 2 hop wavelength paths are pre-configured with wavelength routing for router-level links. However, in the case of the access network, we forbid wavelength paths that

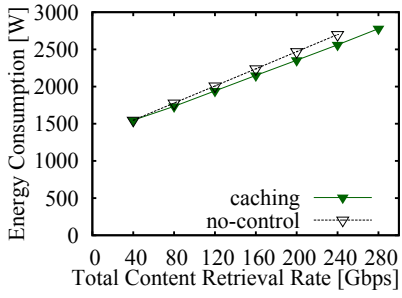

(a) Total content retrieval rate vs. energy consumption

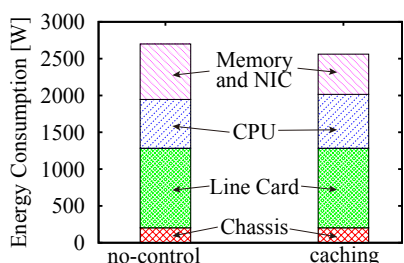

(b) Energy consumed by each component inside routers
Fig. 3: Energy consumed by an access network

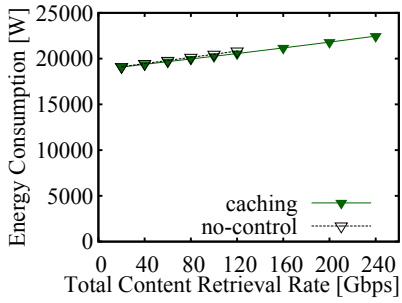

(a) Total content retrieval rate vs. energy consumption

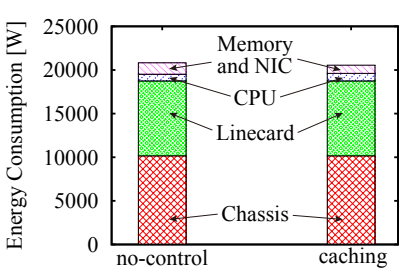

(b) Energy consumed by each component inside routers
Fig. 4: Energy consumed by an core network

directly connect edge routers with the core router. That is, traffic is allowed to be routed from one edge router to another by configuring router-level links with wavelength routing. Note that the results of caching and no-control is the same as that in Figs. 3 and 4 since they cannot cutting off unused links.

Figure 5(a) shows the energy consumed by the entire access network. We have two observations in this result: 1) The effects of cutting off links on the energy reduction decreases as traffic increases. 2) Caching enhances the effects of cutting on the energy reduction. The observation 1) is straightforward since the room for cutting off links decreases as the traffic load increases. Since caching constantly reduces traffic regardless of the traffic load, it also constantly reduces the energy consumption even though the effect is small. In the case that the total content retrieval rate exceed $200 \mathrm{Gbps}$, caching reduces the energy consumption more than cutting. In contrast to 1 ), the observation 2) may seem to be counter-intuitive. For instance, in the case that the total content retrieval rate is 240 Gbps, caching, cutting, and integration reduces the energy consumption by $5.1 \%, 3.4 \%$, and $10.7 \%$ compared with nocontrol. This implies that adding caching to cutting enhances the effects of cutting on the energy reduction.

To investigate the reason why caching enhances the effects of cutting on the energy reduction, we shows the energy consumed by LCs, which is the function of the number of active router-level links, in Fig. 5(b). Caching can never reduce the energy consumed by LCs since it does not change routerlevel links. However, the traffic reduction effect due to caching makes more room to cutting off links and therefore gains the effects of cutting on the energy reduction. Hence, the total energy consumption is reduced by combining caching and 

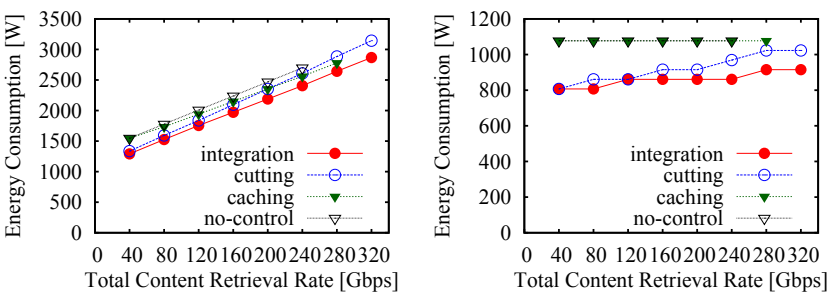

(a) Total content retrieval rate vs. energy consumption of the entire network

(b) Total content retrieval rate vs. energy consumption of LCs

Fig. 5: Energy consumed by an access network with additional router-level links

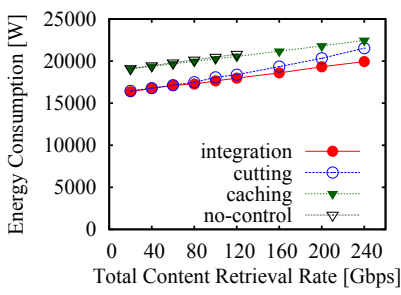

(a) Total content retrieval rate vs. energy consumption of the entire network

Fig. 6: Energy consumed by a core network with additional router-level links

cutting even though caching increases the energy consumed by CPUs. These results may support our hypothesis that caching enhances cutting. The same tendency can be also observed in the case of a core network as shown in Fig. 6.

\section{RELATED WORK}

Several researches focus on the energy reduction by caching. Lee et al. conclude that the reduction of traffic by caching contributes to energy reduction [2]. Choi et al. show that the optimal content placement realizes an energy-efficient ICN [3]. Though they take energy consumed by storage devices for caching contents, they do not consider the energy consumed by other devices, such as CPUs adn NICs, which is not negligible according to the observations in [4].

Other than caching, for the energy reduction of an entire network, Bolla et al. propose two techniques, adaptive rate and low power idle, to reduce energy consumption at routers [16]. These techniques are based on more advanced router architecture, such as dynamic voltage/frequency scaling, than currently available ones, which we assume in the present study. Discussing the caching trade-off assuming such advanced routers is one direction of further study.

\section{CONCLUSION}

Motivated by the research question, whether in-network caching really reduces the energy consumption, we analyzed the effects of caching on the energy reduction. To answer the question, we formulated in-network caching with a realistic energy consumption model for an ICN router as optimization problem that minimizes the energy consumed by the entire network. Through numerical studies, we found the following observations: i) With the ICN forwarding engine currently under development, it is difficult to reduce the energy consumption of the entire network with caching alone because of its tradeoff relation, i.e., the increase and decrease in the energy consumption due to name-based packet forwarding and the traffic reduction. ii) Even though the ICN forwarding engine is not well-optimized, caching enhances the energy reduction effects of a green networking technique, which frees several links by moving their traffic onto other links and cuts off the power of unused links. This implies that improving ICN forwarding engines is one of promising approaches toward energy-efficient ICN networks. These observations are the first step in understanding energy reduction effects.

\section{ACKNOWLEDGEMENTS}

The research leading to these results has partially received funding from the EU-JAPAN initiative by the EC Seventh Framework Programme (FP7/2007-2013) Grant Agreement No. 608518 and NICT under Contract 167 (GreenICN).

\section{REFERENCES}

[1] J. Llorca, A. M. Tulino, K. Guan, J. Esteban, M. Varvello, N. Choi, and D. C. Kilper, "Dynamic in-network caching for energy efficient content delivery," in Proceedings of IEEE INFOCOM, Apr. 2013, pp. 245-249.

[2] U. Lee, I. Rimac, D. Kilper, and V. Hilt, "Toward energy-efficient content dissemination,” IEEE Network, vol. 25, no. 2, pp. 14-19, Mar. 2011.

[3] N. Choi, K. Guan, D. C. Kilper, and G. Atkinson, "In-network caching effect on optimal energy consumption in content-centric networking," in Proceedings of IEEE ICC, Jun. 2012, pp. 2889-2894.

[4] T. Hasegawa, Y. Nakai, K. Ohsugi, J. Takemasa, Y. Koizumi, and I. Psaras, "Empirically modeling how a multicore software ICN router and an ICN network consume power," in Proceedings of ACM ICN, 2014, pp. 157-166.

[5] M. Zhang, C. Yi, B. Liu, and B. Zhang, "GreenTE: Power-aware traffic engineering," in Proceedings of IEEE ICNP, Oct. 2010, pp. 21-30.

[6] S. K. Fayazbakhsh, Y. Lin, A. Tootoonchian, A. Ghodsi, T. Koponen, B. Maggs, K. Ng, V. Sekar, and S. Shenker, "Less pain, most of the gain: incrementally deployable ICN," in Proceedings of ACM SIGCOMM, Aug. 2013, pp. 147-158.

[7] Internet2 Network, http://noc.net.internet2.edu/i2network/ maps-documentation/maps.html.

[8] J. Baliga, R. Ayre, K. Hinton, W. V. Sorin, and R. S. Tucker, "Energy consumption in optical IP networks," Journal of Lightwave Technology, vol. 27, no. 13, pp. 2391-2403, Jul. 2009.

[9] W. So, A. Narayanan, and D. Oran, "Named data networking on a router: Fast and DoS-resistant forwarding with hash tables," in Proceedings of ACM/IEEE ANCS, Oct. 2013, pp. 215-226.

[10] Cisco Systems, Inc., “ASR 9006 Router,” http://www.cisco.com/c/en/us/ products/routers/asr-9006-router/index.html.

[11] Intel Corporation, "Intel ${ }^{\circledR}$ Xeon ${ }^{\circledR}$ Processor 5600/5500 Series Platforms for Embedded Computing," http://www.intel. com/content/dam/www/public/us/en/documents/platform-briefs/ xeon-5500-5600-platform-brief.pdf.

[12] Osaka Prefectural Goverment, http://www.pref.osaka.lg.jp/toukei/top/ kokucho10.html.

[13] U.S. Census Bureau, http://factfinder.census.gov/bkmk/table/1.0/en/PEP/ 2012/PEPANNRSIP.US12A.

[14] C. Fricker, P. Robert, J. Roberts, and N. Sbihi, "Impact of traffic mix on caching performance in a content-centric network," in Proceedings of IEEE NOMEN, Mar. 2012, pp. 310-315.

[15] Project CCNx, http://www.ccnx.org/.

[16] R. Bolla, R. Bruschi, A. Carrega, F. Davoli, D. Suino, C. Vassilakis, and A. Zafeiropoulos, "Cutting the energy bills of internet service providers and telecoms through power management: An impact analysis," Computer Networks, vol. 56, no. 10, pp. 2320-2342, Jul. 2012. 